\title{
OP06
}

\section{MODELLING OF SIMULTANEOUS CONTRAST EFFECTS USING MEMORY COLOUR MATCHING METHOD TO REVISE THE CIECAM16 COLOUR APPEARANCE MODEL}

\section{Yuechen Zhu et al.}

DOI 10.25039/x46.2019.OP06

from

CIE x046:2019

Proceedings

of the

29th CIE SESSION

Washington D.C., USA, June 14 - 22, 2019

(DOI 10.25039/x46.2019)

The paper has been presented at the 29th CIE Session, Washington D.C., USA, June 14-22, 2019. It has not been peer-reviewed by CIE.

(C) CIE 2019

All rights reserved. Unless otherwise specified, no part of this publication may be reproduced or utilized in any form or by any means, electronic or mechanical, including photocopying and microfilm, without permission in writing from CIE Central Bureau at the address below. Any mention of organizations or products does not imply endorsement by the CIE.

This paper is made available open access for individual use. However, in all other cases all rights are reserved unless explicit permission is sought from and given by the CIE.

CIE Central Bureau

Babenbergerstrasse 9

A-1010 Vienna

Austria

Tel.: +43 17143187

e-mail: ciecb@cie.co.at

www.cie.co.at 


\title{
MODELLING OF SIMULTANEOUS CONTRAST EFFECTS USING MEMORY COLOUR MATCHING METHOD TO REVISE THE CIECAM16 COLOUR APPEARANCE MODEL
}

\author{
Zhu, Y. ${ }^{1}$, Luo, M.R. ${ }^{1,2}$ \\ ${ }^{1}$ State Key Laboratory of Modern Optical Instrumentation, Zhejiang University, Hangzhou, \\ CHINA, ${ }^{2}$ School of Design, University of Leeds, Leeds LS2 9JT, UNITED KINGDOM \\ m.r.luo@zju.edu.cn
}

DOI $10.25039 / \times 46.2019 .0 P 06$

\begin{abstract}
Experiments were carried out to investigate the simultaneous colour contrast effect on a selfluminous display using colour matching method. The goals of this study were to build a new visual dataset and to extend CAM16 to include the simultaneous colour contrast effect. Five coloured cubes together with six familiar objects were studied. A total of 325 test/background combinations were displayed on a calibrated display. Two functions based on CAM16 for predicting lightness and hue contrast effects were successfully developed in this study. The model was verified using two data sets, i.e., one was collected in this study including all the familiar objects and two was generated by Wu et al. The present study shows promising results that the contrast effect can be successfully integrated into a colour appearance model.
\end{abstract}

Keywords: Simultaneous Colour Contrast, Colour Appearance Model, Memory Colour Matching

\section{Introduction}

Colour appearance model is used to describe the perception of human colour vision, i.e., predicting the colour appearance under a very wide range of viewing conditions. Over the decades, models have been proposed by various works (Hunt, 1982). The CIE recommended CIECAM02 in 2002, which is widely used as a universal colour model for scientific researches and industrial applications (CIE, 2004a). Recently, CAM16 was proposed to overcome some mathematic problem in CIECAM02 and had a simpler structure (Li et al., 2017). It is in the process to replace CIECAM02 in the CIE.

Simultaneous colour contrast effect and chromatic adaptation are two important visual phenomena for colour appearance model to predict (Luo et al., 1995; CIE, 2004b). Chromatic adaptation refers to the human visual system to adapt to the overall colour and intensity of the illumination to maintain an approximately colour constancy appearance under different illuminants. Meanwhile, simultaneous colour contrast effect causes a shift of colour appearance related to the surrounding colour, and the effect is usually studied in a target-background paradigm (Wu et al., 2004; Wu et al., 2006a; Wu et al., 2006b; Gao et al., 2014). The latter effect has not been modelled by any colour appearance model.

In the previous studies, a series of psychophysical experiments were carried out to investigate the chromatic adaptation using memory colour matching method on a display (Zhu et al., 2018). In this study, the same experimental method was used to study the simultaneous colour contrast effect. Since, the centre patches in target-background paradigm was replaced by images of 2D objects in this study (Smet et al., 2014; Liu et al., 2014). According to previous research, the contrast effect on chroma is very small and partly affected by both the lightness and hue (Gao et al., 2014). The main goal of this study is investigating simultaneous colour contrast effect on hue and lightness (Smet et al., 2011; OLKKONEN et al., 2004; VURRO et al., 2013). The results were used to derive simultaneous contrast function based on CAM16 colour appearance model.

\section{Experimental}

The experiment was conducted on a calibrated EIZO-CG243W display (size: 24.1") in a darkened room. The adapting field on display was set to $6500 \mathrm{~K}$ and $23 \mathrm{~cd} / \mathrm{m} 2\left(L^{*}=50\right)$. 
Observers sat $60 \mathrm{~cm}$ away from the display. The experiment was divided into two parts to investigate the lightness contrast and hue contrast respectively.

\subsection{Experiment 1: simultaneous colour contrast effect on lightness}

In the first part, five cubes showing pure (unitary) red, yellow, green, blue and neutral grey were selected, as shown in Figure 1. Eleven luminance levels of background $\left(L^{*}=0,10,20,30,40\right.$, $50,60,70,80,90$ and 100 ) were selected taking $L^{*}=50$ as the reference. The interface on display was divided into two parts, left and right. Figure 2 shows the experimental setting. The order of left-right arrangements was randomised. One part always included a fixed cube presented on a background of $L^{*}=50$, and the other part had a test cube on test background with a particular $L^{*}$ value. Observers adjusted the lightness of the test object via a keyboard in CIELAB $L^{*}$ scale to match that of the reference cube until they look visually the same, the $\Delta L^{*}$ between each pixel and the mean $L^{*}$ of the whole image was remained. In addition, the hue and chroma of the cubes were fixed, the colour coordinates of that are shown in Table 1. Thirty-two normal colour vision observers ( 17 males and 15 females) took part in the experiment. In total, 1,760 matches were accumulated, i.e., 5 cubes $\times 11$ luminance levels $\times 32$ observers.

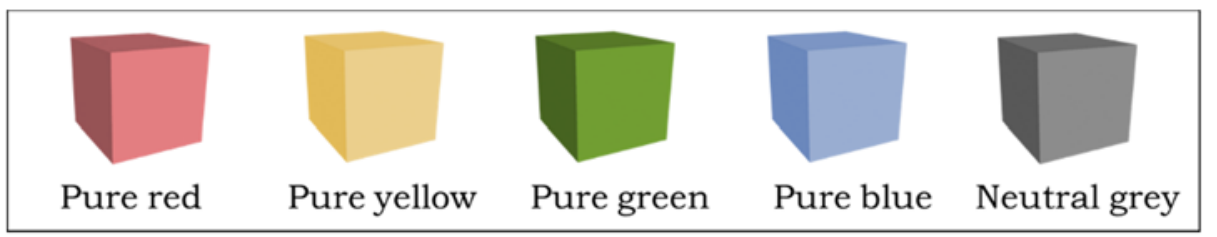

Figure 1 - Images of the five cubes used to perform memory colour matching

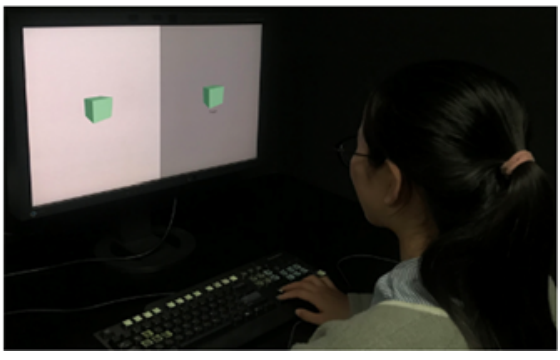

(a)

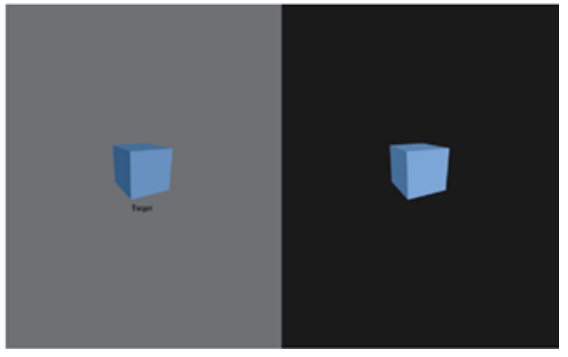

(b)

Figure 2 - Experimental setting. (a) Experimental situation; (b) Operational Interface.

Table 1 - Colour coordinates of the cubes used in the lightness contrast experiment

\begin{tabular}{llllll}
\hline CIELAB & Red & Yellow & Green & Blue & Neutral grey \\
\hline$a^{*}$ & 29.0 & 5.2 & -26.4 & -10.3 & 0.4 \\
\hline$b^{*}$ & 3.4 & 28.9 & 12.9 & -27.7 & -2.3 \\
\hline$h$ & 29.2 & 29.3 & 29.4 & 29.5 & 2.3 \\
\hline$C^{*}$ & 6.7 & 79.7 & 154.0 & 249.6 & 279.6 \\
\hline
\end{tabular}

\subsubsection{Observer variation}

Mean Colour Difference from the Mean (MCDM) was calculated to represent the observer variation of the result. The overall MCDM value was found to be 2.1 and 3.2 of $\Delta E^{\prime}$ Jab units for intra- and inter- observer variation respectively, which were comparable to similar studies. Figure 3 shows the average MCDM of each object for characterizing the inter-observer variations. 


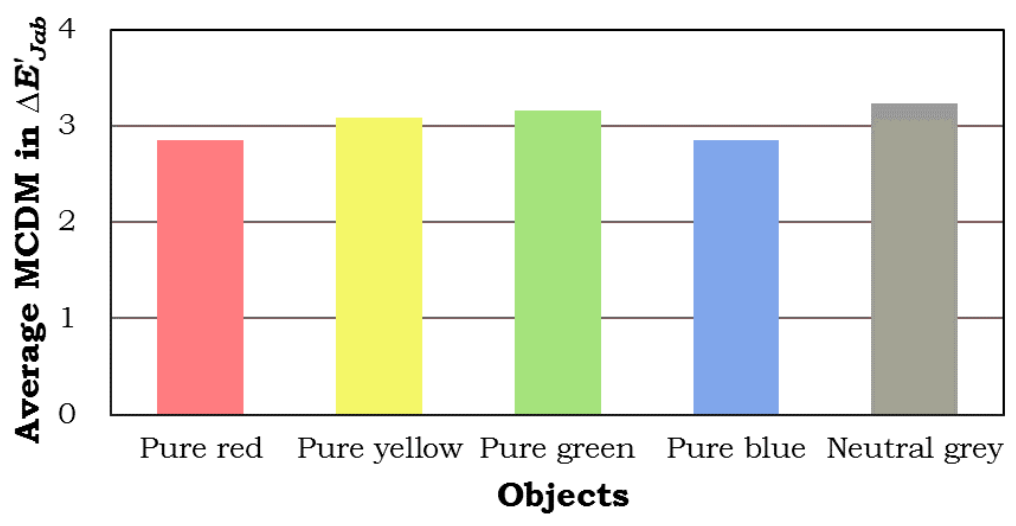

Figure 3 - The average MCDM of each object for characterizing the inter-observer variations

\subsubsection{Average results of each colour}

The adjusted results were calculated in CAM16 colour space. The result was plotted by $\Delta J_{b-r}^{\prime}$ vs $\Delta J^{\prime} v$, where $\Delta J^{\prime} b$-r was the lightness differences between test against background and the test against reference background $\left(L^{*}=50\right)$. The differences in lightness $\Delta J^{\prime} v$ represents the visual lightness shifts between the results of test/background and the test/reference $\left(L^{*}=50\right)$. Figure 4 shows the average results of the five objects adjusted by the observers, the colour coding scheme of the dots corresponding to the target colour of the series, i.e., Pure red, Pure yellow, Pure green, Pure blue and Neutral grey. It can be seen that a clear relationship was clearly discerned for all colour centres.

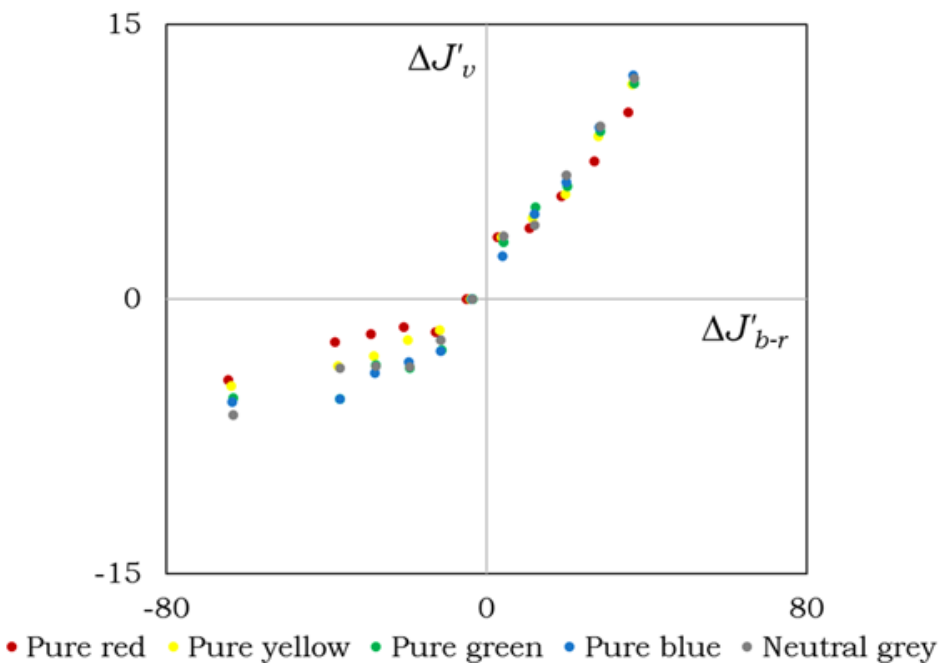

Figure 4 - The average results of the five objects adjusted by the observers

\subsection{Experiment 2: simultaneous colour contrast effect on hue}

In the second part, ten objects including six familiar objects (Red apple, Sky, Green pepper, Eggplant, Banana and Tomato) and four coloured cubes from the first part (Red, Yellow, Green, Blue) were selected. Figure 5 shows the images of the familiar objects. Each object was against three backgrounds differed in size $\left(7^{\circ}, 15^{\circ}\right.$ and $\left.25^{\circ}\right)$ together with no background (the whole screen is grey, as a reference ). Also, each size of background was set to eight colours $(h \pm 45$, $h \pm 30, h \pm 20$ and $h \pm 10$; where $h$ is the CIELAB hue angle of the object).

Sixty normal colour vision observers (26 males and 34 females) took part in the experiment. And the colour of each object was matched by 20 observers. After one minutes' adaptation, observers viewed a grey image of one object with a particular size of coloured background and adjusted the chroma and hue of the object to match their memory colour using a keyboard in CIELAB space (as for cubes, only adjusted the hue of the objects). The lightness was fixed to remain the image information (texture and gloss). Figure 6 shows the experimental setting. In 
total, 5,400 matches were accumulated, i.e., 10 objects $\times 3$ sizes of background $\times$ ( 8 coloured backgrounds +1 no background) $\times 20$ observers.

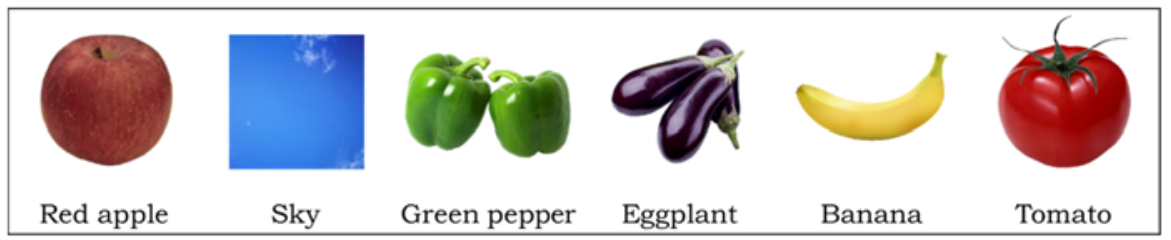

Figure 5 - Images of the six familiar objects used to perform memory colour matching

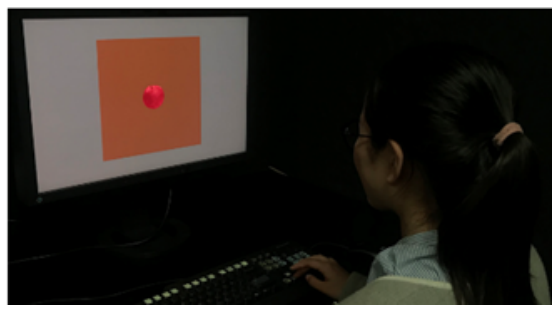

(a)
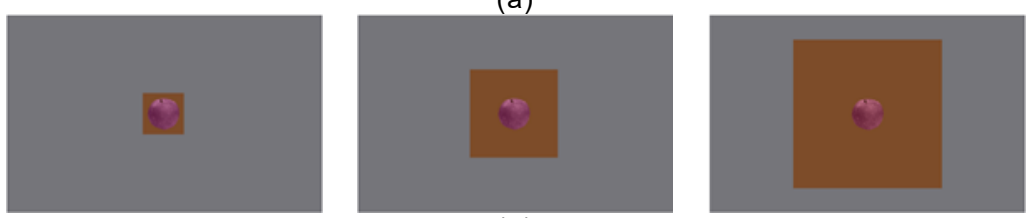

(b)

Figure 6 - Experimental setting. (a) Experimental situation; (b) Operational Interface, background: $7^{\circ}, 15^{\circ}, 25^{\circ}$.

\subsubsection{Observer variation}

Mean Colour Difference from the Mean (MCDM) was calculated to represent the observer variation of the result. The overall MCDM value was found to be 2.0 and 2.5 of CAM16 $\Delta E^{\prime}$ Jab units for intra- and inter- observer variation respectively, which were comparable to similar studies. Figure 7 shows the average MCDM of each object for characterizing the inter-observer variations.

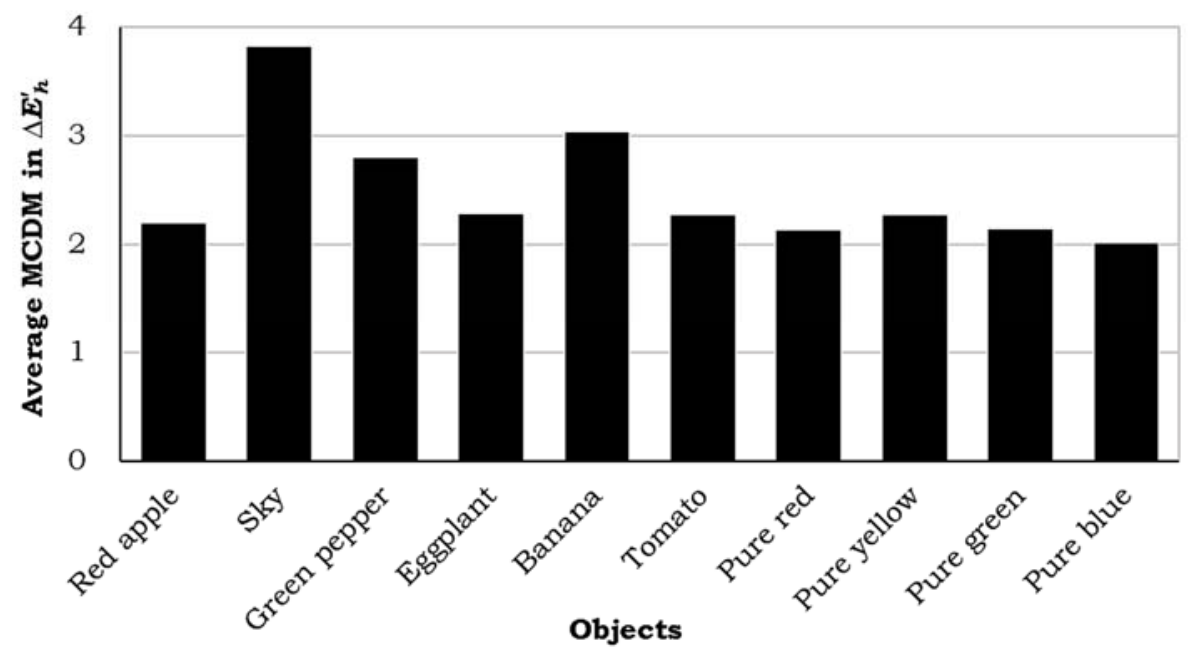

Figure 7 - The average MCDM of each object for characterizing the inter-observer variations

\subsubsection{Average results of each colour}

As mentioned earlier, taking test/grey background (no background) as a reference, the differences in hue angle $\Delta h^{\prime}$, representing the visual colour shifts between the results of test/background and the test/grey background (reference). The differences in hue angle $\Delta h_{b-r}^{\prime}$ 
was the colour differences between coloured background and target colour (the result of test/grey background). All the parameters were calculated again using CAM16.

The hue metric differences $\Delta H_{v}^{\prime}$ and $\Delta H_{b-r}^{\prime}$ were determined from the hue angle differences $\Delta h_{v}^{\prime}$ and $\Delta h_{b-t}^{\prime}$, using Equation (1) to make the same visual scale as the lightness and chroma differences for all the combinations. The hue contrast has not been considered in the CAM16, so the model is expected to give a poor performance. Taking the results of cubes as an example, Figure 8 shows the average results of the four cubes adjusted by the observers, the colour coding scheme of the dots corresponding to the size of the backgrounds, i.e., $7^{\circ}, 15^{\circ}$ and $25^{\circ}$. It can be found that a sinusoidal relationship was discerned, and the size of the background had little impact on the results. So the modelling was based on all the data of three size of backgrounds described in Section 3.1.

$$
\Delta H=2 \times\left(C_{1} C_{2}\right)^{0.5} \sin \left(\frac{\Delta h}{2}\right)
$$

where $C_{1}$ and $C_{2}$ are the CAM16-UCS chroma.

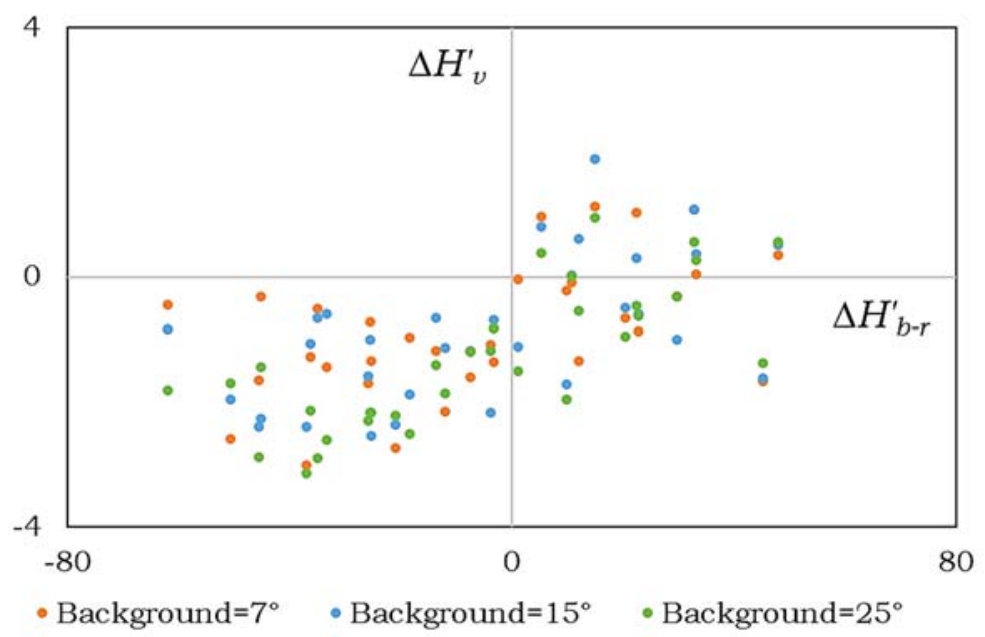

Figure 8 - The average results of the cubes of unitary hues

\section{Modelling the simultaneous contrast effect in CAM16}

The model was based on the data of cubes to modified the CAM16. In the computation, lightness contrast is performed first and then followed by the hue contrast. Figure 9 shows the flow chart of the modelling. Note that chroma contrast effect was not studied. From the Gao et al.'s results (Gao et al., 2014), they concluded that the chroma effect is much smaller than the lightness and hue contrast effect, and chroma effect is partly affected by both the lightness and hue. So, it is not studied here.

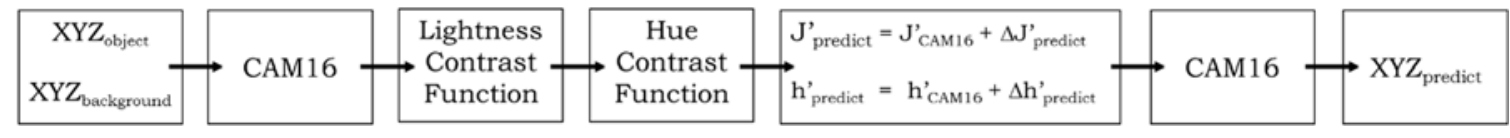

Figure 9 - The flow chart of the modelling

\subsection{Lightness simultaneous contrast effect}

To reveal the contrast effect, the visual results were expressed as each test/background combination. A hyperbolic function was fitted and give accurate prediction to the data, as given in Equation (2):

$$
\Delta J_{\text {predict }}^{\prime}=\frac{9.17}{0.36+e^{-0.05 \times \Delta J_{b-r}^{\prime}}}-5.80
$$


Figure 10 shows the curve of the lightness contrast function with a clear trend, i.e., a darker background will make target brighter. The data were used to test CAM16 original and refined models, and the results showed that the lightness contrast function improved $R$ from 0.12 to 0.98 and reduced prediction error $\left(\Delta J^{\prime}\right)$ from 5.91 to 0.95 . Note that CAM16 already included function to consider the lightness contrast effect, but it does not work well.

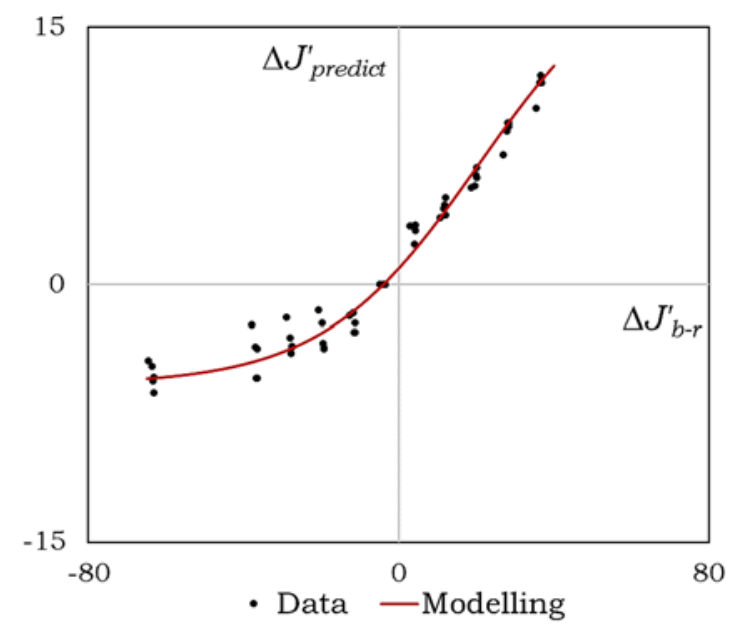

Figure 10 - The curve of the lightness contrast function fitted the data

\subsection{Hue simultaneous contrast effect}

A sine function was well fitted to consider both the size and hue of the background. Each equation modelled the contrast effect by showing a transition at the turning point of zero (representing the lightness or hue composition of the target). In its both sides, the sign of colour difference changes from negative to positive. The trend was modelled by a sigmoidal function, as given in Equation (3):

$$
\Delta H_{\text {predict }}^{\prime}=0.0833 \times \Delta H_{b-r}^{\prime} \times e^{-0.001 \times\left(\Delta H^{\prime}{ }_{b-r}\right)^{2}}
$$

Figure 12 shows the curve of the hue contrast function. The data were used to test CAM16 original and refined models, the results showed that the hue contrast function improved $R$ from 0.00 to 0.67 and reduced prediction error $\left(\Delta H^{\prime}\right)$ from 1.5 to 1.2. Note that CAM16 does not consider hue contrast effect so that $R$ was equal to zero. Thus, by hue contrast, the model shows a big improvement.

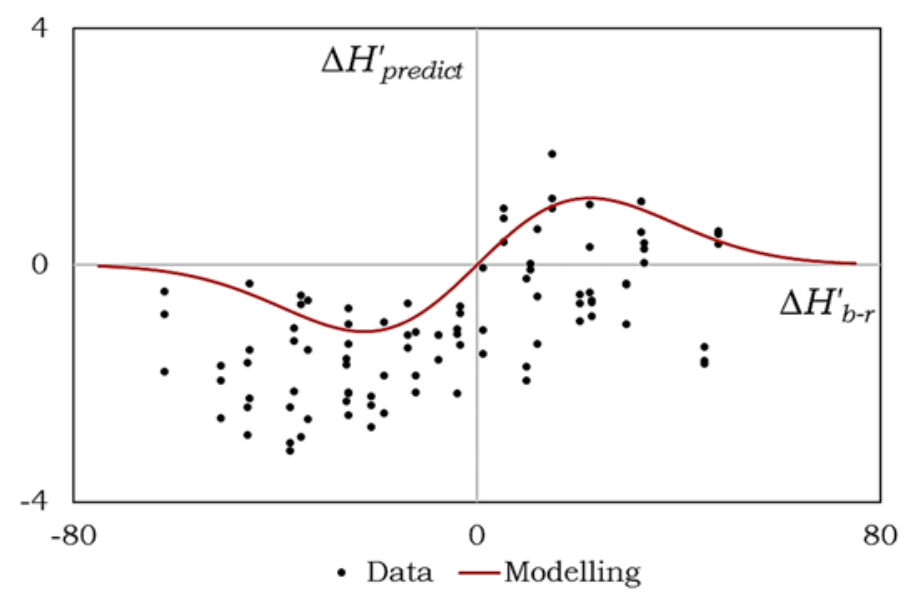

Figure 11 - The data points and prediction results of the hue contrast function 


\subsection{Testing the refined model}

Both contrast functions were added to CAM16 to form a refined model. The model was verified using two data sets, i.e., one was collected in Experiment 2 including all the familiar objects and the other was generated by Wu et al. using the magnitude estimation method on patches.

\subsubsection{The data of the familiar objects collected in Experiment 2}

Figure 12 shows the predictive performance of the refined model to fit the familiar objects collected in Experiment 2. It can be seen that the data were very scatterd, but the model predicts the trend to some extent. The colour difference of prediction is 2.09 of $\Delta H^{\prime}$ units. This implies that the memory colour is stable and quite exempt from the influence of contrast effect. Thus, the contrast effect is influenced by the familarity of the objects to a degree.

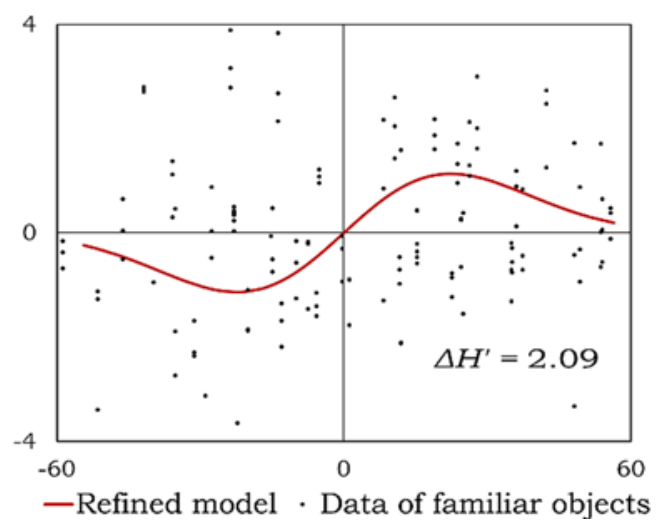

Figure 12 - The predictive performance of the refined model to fit the familiar objects collected in Experiment 2

\subsubsection{The Wu et al.'s data (Wu et al., 2004; Wu et al., 2006b)}

The Wu et al.'s data were obtained from a series of psychophysical experiments. The experimental settings were under well controlled conditions and the testing colours were selected carefully. The results showed a clear trend with high reliability. It is also used here as an independent test dataset. Figure 13 shows the predictive performance of the refined model to fit the Wu's data. It can be found that the model predicts well to the trend both in lightness and hue. The colour differences of prediction were 2.45 of $\Delta J^{\prime}$ units and 0.83 of $\Delta H^{\prime}$ units respectively.

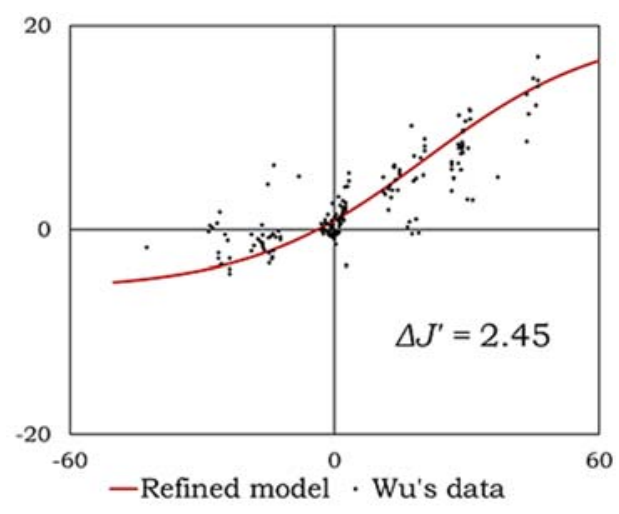

(a)

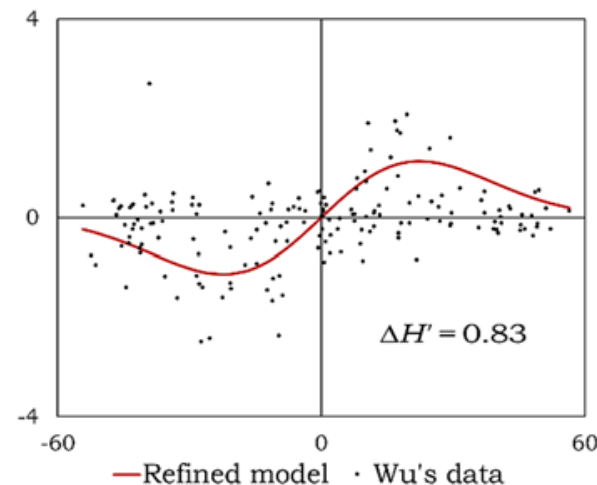

(b)

Figure 13 - The predictive performance of the refined (a) the lightness contrast and b) hue contrast model to fit the Wu's data

In general, the model predicts contrast effect accurately, but the extent of the hue contrast is very much depending on the experimental technique, i.e., a much smaller effect was found from those based on familiar objects with a long-term memory. This implies that the objects selection, experimental technique could have a big impact on hue contrast effect. 


\section{Conclusion}

Two functions based on CAM16 for predicting lightness and hue contrast effects were successfully developed in this study. The model was verified using two data sets, i.e., one was collected in this study including all the familiar objects and two was generated by Wu et al. It gave accurate prediction to the lightness visual results. For hue contrast, it was found a consistent trend. However, the function predicts too low contrasts for the familiar objects by memory matching. This implies that there is a clear colour contrast effect but that is influenced by the familiarity of the objects to a degree, i.e., the memory colour is stable and quite exempt from the influence of contrast effect.

The present study shows promising results that the contrast effect can be successfully integrated into a colour appearance model.

\section{Acknowledgement}

The work is supported by National Natural Science Foundation of China (Grant number: $61775190)$.

\section{References}

HUNT, R.W.G. 1982. A Model of Colour Vision for Predicting Colour Appearance. Color Research \& Application, 7(2), 95-112.

CIE 2004. CIE 159:2004. A Colour Appearance Model for Colour Managment Systems: CIECAM02. Vienna: CIE.

LI, C.J., LI, Z., WANG, Z., XU, Y., LUO, M.R., CUI, G.H., MELGOSA, M., BRILL, M.H., POINTER, M.R. 2017. Comprehensive Color Solutions, CAM16, CAT16 and CAM16-UCS. Color Research \& Application, 42(6), 703-718.

LUO, M.R., GAO, X.W. 1995. Quantifying Colour Appearance. Part V. Simultaneous Contrast. Color Research \& Application, 20(1), 18-28.

CIE 2004. CIE 160:2004. A Review of Chromatic Adaptation Transforms. Vienna: CIE.

WU, R.C., WARDMAN, R.H., LUO, M.R. 2004. A Comparison of Lightness Contrast Effects in CRT and Surface Colours. Color Research \& Application, 30(1), 13-20.

WU, R.C., WARDMAN, R.H. 2006. Lightness and Hue Contrast Effects in Surface (Fabric) Colours. Color Research \& Application, 32(1), 55-64.

WU, R.C., WARDMAN, R.H. 2006. Proposed Modification to the CIECAM02 Colour Appearance Model to Include the Simultaneous Contrast Effects. Color Research \& Application, 32(2), 121-129.

GAO, X.W., WANG, Y., QIAN, Y., GAO, A. 2014. Modelling of Chromatic Contrast for Retrieval of Wallpaper Images. Color Research \& Application, 40(4), 361-373.

ZHU, Y., LUO, M.R., ZHAI, Q. 2018. Investigating Chromatic Adaptation via Memory Colour Matching Method on a Display. Color and Imaging Conference, 313-317.

SMET, K.A.G., LIN, Y., NAGY, B.V., NEMETH, Z., DUQUE-CHICA, G.L., QUINTERO, J.M., CHEN, H.S., LUO, M.R., SAFI, M., HANSELAER, P. 2014. Cross-culture Variation of Memory Colors of Familiar Objects. Optics Express, 22(26), 32308-32328.

LIU, X.Y., LUO, M.R. 2014. Investigating Observer Variability for Assessing Memory Colours. Color and Imaging Conference, 200-205.

SMET, K.A.G., RYCKAERT, W.R., POINTER, M.R. 2011. Colour Appearance Rating of Familiar Real Objects. Color Research \& Application, 36(3),192-200.

OLKKONEN, M., HANSEN, T., GEGENFURTNER, K.R. 2004. Color Appearance of Familiar Objects: Effects of Object Shape, Texture, and Illumination Changes. Journal of Vision, 8(5), 1-16.

VURRO, M., LING, Y., HURLBERT, A.C. 2013. Memory Color of Natural Familiar Objects: Effects of Surface Texture and 3-D Shape. Journal of Vision, 13(7), 1-20. 